Research Article

\title{
Age-Related Change of Axial Length, Spherical Equivalent, and Prevalence of Myopia and High Myopia in School-Age Children in Shanghai: 2014-2018
}

\author{
Tao Li $\mathbb{D i D}^{1,2}$ Bo Jiang $(\mathbb{D})^{1}$ and Xiaodong Zhou ${ }^{1}{ }^{1}$ \\ ${ }^{1}$ Department of Ophthalmology, Jinshan Hospital of Fudan University, Shanghai, China \\ ${ }^{2}$ Department of Ophthalmology, Eyed ENT Hospital of Fudan University, Shanghai, China \\ Correspondence should be addressed to Xiaodong Zhou; xdzhou2005@163.com
}

Received 2 May 2020; Revised 30 October 2020; Accepted 30 October 2020; Published 12 November 2020

Academic Editor: Alessandro Meduri

Copyright (C) 2020 Tao Li et al. This is an open access article distributed under the Creative Commons Attribution License, which permits unrestricted use, distribution, and reproduction in any medium, provided the original work is properly cited.

Purpose. To investigate the age-related change of axial length (AL), spherical equivalent (SE), and prevalence of myopia and high myopia in children at 7-18-year-olds in Shanghai in 2014 and 2018, respectively. Methods. This was an observational study in Shanghai. The same 3 schools were selected in 2014 and 2018, respectively. AL, SE, prevalence of myopia and high myopia, height, and weight were measured. A questionnaire regarding the lifestyles was completed. Results. Mean age was smaller in 2018 than in $2014(P<0.001)$, and mean AL was shorter in 2018 than in $2014(P=0.003)$, whereas mean SE was greater in 2018 than in 2014 $(P<0.001)$. The prevalence of myopia and high myopia was lower in 2018 than in $2014(P<0.001$ and $P=0.013$, respectively). Mean AL increased with age from 7-year-olds to 18-year-olds in 2014 and 2018 (both $P<0.001$ ), respectively. Mean SE decreased with age in 2014 and 2018 (both $P<0.001$ ), respectively. The prevalence of myopia and high myopia increased with age in 2014 and 2018 (all $P<0.001$ ), respectively. Less mean time outdoors and more mean time of study of all children were observed in 2018 than in 2014 ( $P=0.018$ and $P<0.001$, respectively). Conclusion. This study shows normative growth values for AL and SE in Shanghai children at the age of 7-18-year-olds, as well as the age-specific prevalence of myopia and high myopia.

\section{Introduction}

Myopia is the most common eye disorder worldwide [1-3], which is mainly caused by a mismatch between the optical power of the eye and its excessively long axial length (AL). The correction modalities for myopia include spectacle lenses [4], contact lenses [5], photorefractive keratectomy [6], laser epithelial keratomileusis [7], laser in situ keratomileusis (LASIK) [7], femtosecond laser-assisted LASIK [8], small-incision lenticule extraction (SMILE) [8], and Visian implantable collamer lens (ICL) implantations [9]. However, due to the excessive axial elongation, high myopia can increase the risk of visual impairment complications (e.g., retinal detachment, maculopathy, and glaucoma) $[1,10,11]$, which can lower life satisfaction and reduce the quality of life $[12,13]$. Thus, the control of myopia progression has become very essential.
Although the mechanism of myopia development is still unclear, myopia is the result of genetic and environmental factors. The prevalence of myopia and high myopia is relatively high in East and Southeast Asian regions [14-17], partly due to increasing environment risk factors. The changing lifestyles (e.g., time outdoors and time of study) $[17,18]$ and classical risk factors (e.g., education $[19,20]$ and ethnicity [3]) are associated with the increased risk of progression of myopia in school-age children. Updating the age-related change trends of myopia-related data in childhood is very important for myopia prevention and controlling. We conducted this study to investigate age-related change trends for AL, SE, and prevalence of myopia and high myopia in children at 7-18-year-olds in Shanghai in 2014 and 2018, respectively. In addition, we also investigated whether these differences between 2014 and 2018 could be explained by the time outdoors and time of study. 


\section{Methods}

2.1. Subjects. This was an observational study in Jinshan District, Shanghai, China. One primary school, one junior high school, and one senior high school were randomly selected in 2014, and the same schools were studied in 2018. The exclusion criteria were a history of severe systemic and ocular diseases (e.g., cataract, glaucoma), topical application of low-concentration atropine eye drops, and orthokeratology lens correction. The study children were divided to three groups: primary school group (7-11-year-olds), junior high school group (12-15-year-olds), and senior high school group (16-18-year-olds).

The study was approved by the Ethics Committee of Jinshan Hospital of Fudan University, China. All study procedures adhered to the tenets of the Declaration of Helsinki. Written informed consent was obtained from the parents or guardians of all children.

2.2. Examination. The children's ocular examinations were completed from October 1 to December 31 in 2014 and from September 1 to October 31 in 2018, respectively. Axial length (AL) was obtained using an ocular biometry system (IOL Master; Carl Zeiss Meditec, Oberkochen, Germany). According to our previous studies [21, 22], refraction was measured using an autorefractor (RK-F1; Canon Corporation, Tokyo, Japan) without cycloplegia. Body height and weight were recorded for all children. The height and weight were measured in a standard manner without shoes and thick clothes.

Furthermore, a questionnaire regarding the daily lifestyles was completed by the child's parents or guardians when the child started school in September. Time outdoors and time of study were obtained using questions such as "How much time does your child spend outdoors every day?" and "How much time does your child spend in study every day?" separately for weekdays and weekend days, respectively. The mean number of hours per day was calculated as time spent during weekdays $\times 5+$ time spent in weekend days $\times 2 / 7$.

2.3. Statistical Analysis. Both eyes of each child were examined, and only data from the right eye was used for analysis. Spherical equivalent (SE) was calculated as the sum of sphere power and half of cylinder power. Myopia was defined as $\mathrm{SE} \leq-0.50 \mathrm{D}$ and high myopia as $\mathrm{SE} \leq-6.00 \mathrm{D}$ [23]. Furthermore, high myopia was also analyzed using the definition of AL > $26 \mathrm{~mm}$ [24].

SPSS V.17.0 software was used for data analysis. The independent $t$-test was used to compare the differences of age, AL, and SE between 2014 and 2018. One-way analysis of variance with the Bonferroni post hoc test was used to analyze the differences of age, AL, and SE in 2014 and 2018, respectively. The chi-square test was used to analyze the differences of prevalence of myopia and high myopia in 2014 and 2018, respectively, and to compare the differences between 2014 and 2018. All $P$ values were two-sided and considered statistically significant when less than 0.05 .

\section{Results}

The characteristics for each age group in 2014 and 2018 are shown in Table 1 . A total of 3057 children were examined in 2014 and 3658 children in 2018. Among them, there were 1478 (48.3\%) and 1817 (49.7\%) girls in 2014 and 2018, respectively. The numbers of girls in 2014 and 2018 were smaller than of boys in primary school and junior high school, whereas the numbers of girls in 2014 and 2018 was greater than the number of boys in senior high school.

The comparisons of age, AL, and SE of children between 2018 and 2014 are shown in Table 2. Mean age of all the children was smaller in 2018 than in $2014(P<0.001)$. Children of primary school, junior high school, and senior high school were younger in 2018 than in $2014(P<0.001$, $P<0.001$, and $P=0.049$, respectively). Mean AL of all children was shorter in 2018 than in $2014(P=0.003)$. However, no significant differences were found in children of primary school, junior high school, and senior high school between 2018 and 2014 (all $P>0.05$ ). Further analysis showed that boys had shorter AL in 2018 than in 2014 $(24.17 \pm 1.34 \mathrm{~mm}$ vs. $24.05 \pm 1.30 \mathrm{~mm}, P=0.009)$, and no significant difference was found in girls between 2018 and $2014(23.72 \pm 1.35 \mathrm{~mm}$ vs $23.65 \pm 1.31 \mathrm{~mm}, P=0.160)$. In 2014, mean AL increased from $22.54 \pm 0.65 \mathrm{~mm}$ at 7 -yearolds to $25.05 \pm 1.26 \mathrm{~mm}$ at 18 -year-olds $(P<0.001$, Figure 1(a)), and in 2018, mean AL increased from $22.64 \pm 0.72 \mathrm{~mm}$ to $25.09 \pm 1.20 \mathrm{~mm}$ across this same period $(P<0.001$, Figure 1(a)). Mean ALs of 9- and-11-year-old children were longer in 2018 than in $2014(P=0.001$ and $P=0.001$, respectively). Furthermore, mean SE of all the children was less negative in 2018 than in $2014(P<0.001)$. However, no significant differences were found in children of primary school, junior high school, and senior high school between 2018 and 2014 (all $P>0.05$ ). In 2014, mean SE decreased from $+0.09 \pm 0.78 D$ at 7 -year-olds to $-3.97 \pm 2.30$ $D$ at 18 -year-olds $(P<0.001$, Figure $1(\mathrm{~b}))$, and in 2018 , mean SE decreased from $+0.07 \pm 0.87 D$ to $-4.07 \pm 2.51 D$ across this same period $(P<0.001$, Figure $1(b))$. Mean SEs of $11-$ and 15-year-old children were more negative in 2018 than in 2014 ( $P=0.036$ and $P=0.003$, respectively), whereas mean SE of 17-year-old children was greater in 2018 than in 2014 $(P=0.043)$.

The comparisons of prevalence of myopia and high myopia of children between 2018 and in 2014 are also shown in Table 2. The prevalence of myopia in all the children was lower in 2018 than in $2014(P<0.001)$. However, the prevalence of myopia in junior high school was higher in 2018 than in $2014(P<0.001)$, and no significant differences between 2018 and 2014 were found in children of primary school and senior high school $(P=0.510$ and $P=0.519$, respectively). As illustrated in Figure 2, the prevalence of myopia increased from $17.6 \%$ at 7 -year-old children to 93.3\% at 18 -year-olds in $2014(P<0.001)$ and from $15.7 \%$ to $93.6 \%$ across this same period in $2018(P<0.001)$. Only the prevalence of myopia of 11-year-old children was higher in 2018 than in 2014 (63.3\% vs. 54.2\%, $P=0.039)$. No significant difference between 2018 and 2014 was observed in the prevalence of high myopia defined by $S E \leq-6.00 \mathrm{D}$ in all 
TABLE 1: Characteristics for each age group in 2014 and 2018.

\begin{tabular}{|c|c|c|c|c|c|}
\hline & \multirow{2}{*}{ Age (years) } & \multicolumn{2}{|c|}{2014} & \multicolumn{2}{|c|}{2018} \\
\hline & & $N$ & Girls (\%) & $N$ & Girls (\%) \\
\hline \multirow{6}{*}{ Primary school } & 7 & 250 & $132(52.8)$ & 428 & $202(47.2)$ \\
\hline & 8 & 271 & $135(49.8)$ & 451 & $207(45.9)$ \\
\hline & 9 & 256 & $119(46.5)$ & 380 & $178(46.8)$ \\
\hline & 10 & 252 & $115(45.6)$ & 275 & $136(49.5)$ \\
\hline & 11 & 247 & $110(44.5)$ & 264 & $135(51.1)$ \\
\hline & Total & 1276 & $611(47.9)$ & 1798 & $858(47.7)$ \\
\hline \multirow{5}{*}{ Junior high school } & 12 & 233 & $114(48.9)$ & 315 & $152(48.3)$ \\
\hline & 13 & 230 & $109(47.4)$ & 251 & $122(48.6)$ \\
\hline & 14 & 189 & $81(42.9)$ & 169 & $76(45.0)$ \\
\hline & 15 & 175 & $63(36.0)$ & 149 & $62(41.6)$ \\
\hline & Total & 827 & $367(44.4)$ & 884 & $412(46.6)$ \\
\hline \multirow{4}{*}{ Senior high school } & 16 & 299 & $158(52.8)$ & 338 & $194(57.4)$ \\
\hline & 17 & 328 & $173(52.7)$ & 343 & $189(55.1)$ \\
\hline & 18 & 327 & $169(51.7)$ & 295 & $164(55.6)$ \\
\hline & Total & 954 & $500(52.4)$ & 976 & $547(56.0)$ \\
\hline
\end{tabular}

children $(P=0.155)$. However, the prevalence of high myopia in junior high school was higher in 2018 than in 2014 $(P=0.030)$, and no significant differences between 2018 and 2014 were found in children of primary school and senior high school $(P=1.000$ and $P=0.378$, respectively). As illustrated in Figure 3(a), the prevalence of high myopia increased from $1.6 \%$ at 11 -year-olds children to $19.3 \%$ at 18 year-olds in $2014(P<0.001)$ and from $0.2 \%$ at 7 -year-olds children to $21.4 \%$ at 18 -year-olds in $2018(P<0.001)$. Only the prevalence of high myopia of 14-year-olds children was higher in 2018 than in 2014 ( $7.7 \%$ vs. $2.6 \%, P=0.029)$. The prevalence of high myopia (defined by $\mathrm{AL}>26 \mathrm{~mm}$ ) was lower in 2018 than in $2014(P=0.013)$. However, no significant difference was observed in the prevalence of high myopia in children of primary school, junior high school, and senior high school between 2018 and 2014 (all $P>0.05$ ). As illustrated in Figure 3(b), the prevalence of high myopia increased from $1.2 \%$ at 10 -year-olds children to $20.8 \%$ at 18 year-olds in $2014(P<0.001)$ and from $0.3 \%$ at 9 -year-olds children to $21.7 \%$ at 18 -year-olds in $2018(P<0.001)$.

The comparisons of the time outdoors and time of study of children between 2018 and 2014 are shown in Table 2 as well. Mean time outdoors of all children was less in 2018 than in $2014(P=0.018)$. Further analysis showed that mean time outdoors in children of primary school was less in 2018 than in $2014(P<0.001)$, but no significant differences between 2018 and 2014 were found in mean time outdoors in children of junior high school and senior high school ( $P=0.065$ and $P=0.057$, respectively). Mean time of the study of all children was more in 2018 than in 2014 $(P<0.001)$. Further analysis showed that the mean time of study in children of primary school, junior high school, and senior high school was more in 2018 than in 2014 (all $P<0.001)$.

\section{Discussion}

The present study showed the increase of AL and decrease of $\mathrm{SE}$, as well as the increase of prevalence of myopia and high myopia with age in children at 7-18-year-olds in Shanghai in 2014 and 2018, respectively. Furthermore, age, AL, and prevalence of myopia and high myopia were smaller in 2018 than in 2014, whereas SE was greater in 2018 than in 2014. In addition, mean time outdoors was less in 2018 than in 2014, whereas the mean time of study was more in 2018 than in 2014.

In this study, the positive trends for $\mathrm{AL}$ reflected the continued growth of the eye from 7-18-year-olds, with an increase of approximately $0.228 \mathrm{~mm} /$ year in 2014 and $0.222 \mathrm{~mm} /$ year in 2018, respectively. Similar trends were observed in previous studies. Xiong et al. [25] found that AL increased from an average of $22.87 \mathrm{~mm}$ at 7 -year-olds to $25.50 \mathrm{~mm}$ at 18-year-olds in Shanghai, with an increase of approximately $0.239 \mathrm{~mm} /$ year. Lu et al. [26] found that AL increased from an average of $22.76 \mathrm{~mm}$ at 7 -year-olds to $24.50 \mathrm{~mm}$ at 18-year-olds in Shandong, with an increase of approximately $0.158 \mathrm{~mm} /$ year. Tideman et al. [27] found that mean ALs in European children were $22.36 \mathrm{~mm}$ at 6year-olds, $23.10 \mathrm{~mm}$ at 9-year-olds, $23.41 \mathrm{~mm}$ at 15 -yearolds, respectively, with increase of approximately $0.117 \mathrm{~mm} /$ year. However, there were differences in AL and the increase rate of $\mathrm{AL}$ among different studies. For example, $\mathrm{AL}$ of $25.09 \pm 1.20 \mathrm{~mm}$ at 18 -year-olds and mean increase rate of AL $0.222 \mathrm{~mm} /$ year in 2018 in this study were smaller than the finding of Xiong and coworkers [25], but greater than the finding of $\mathrm{Lu}$ and coworkers [26]. In comparison of AL and its increase rate among these studies, the differences in the genetic and socioeconomic background should be noted, which was not taken into account in this study. Furthermore, the differences in $\mathrm{AL}$ may be related to $\mathrm{AL}$ measurement techniques (IOL Master [25-27] and immersion A-scan sonogram [27]). In addition, the increase rate of AL may be associated with age range, which was greater in this study (11-year-olds) than Tideman et al. [27] (9-year-olds).

In this study, SE was negatively associated with AL and decreased with time, with a decrease of approximately 0.37 D/year in 2014 and $0.38 \mathrm{D} /$ year in 2018, respectively, which was consistent with other studies. Xiong et al. [25] found that 


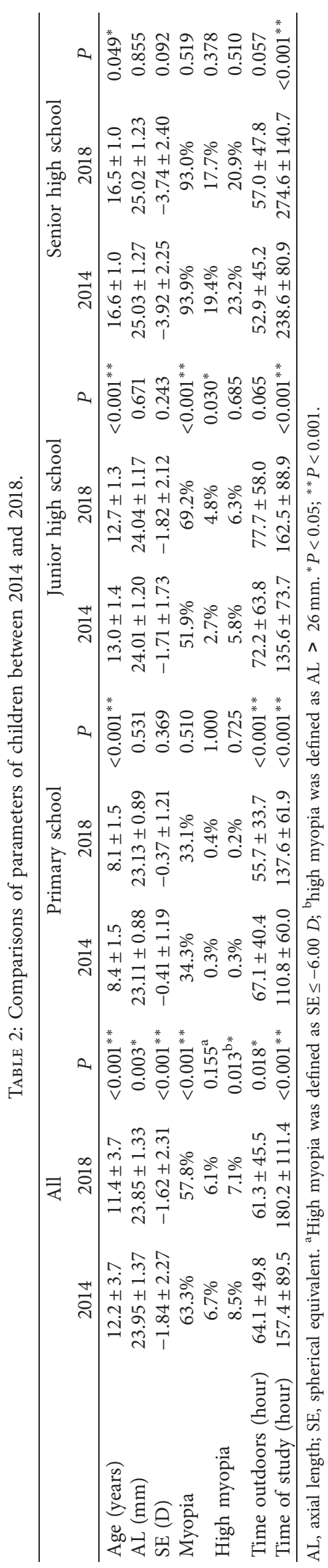




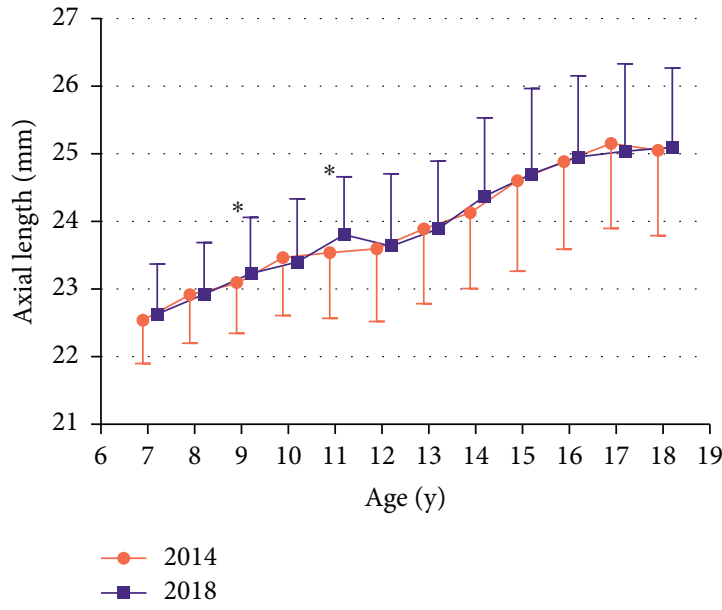

(a)

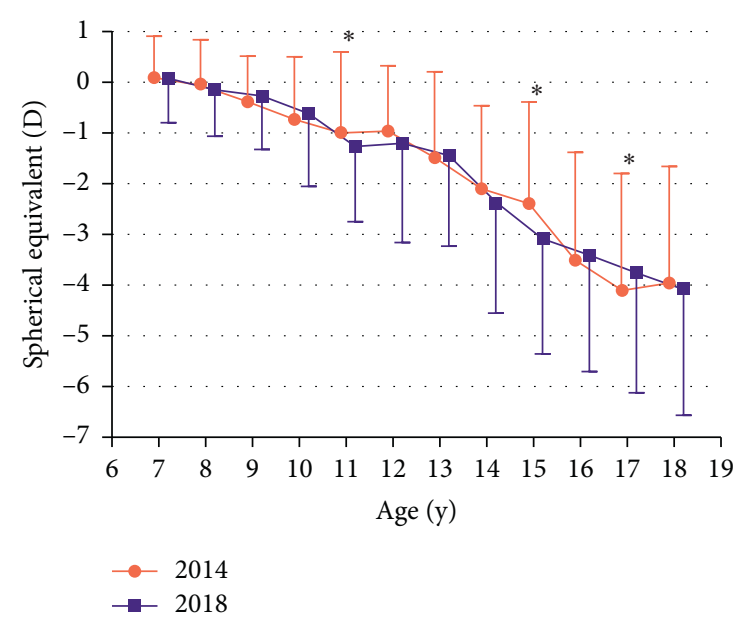

(b)

Figure 1: Trends of axial length (a) and spherical equivalent (b) in children from 7- to 18-year-olds in 2014 and 2018.

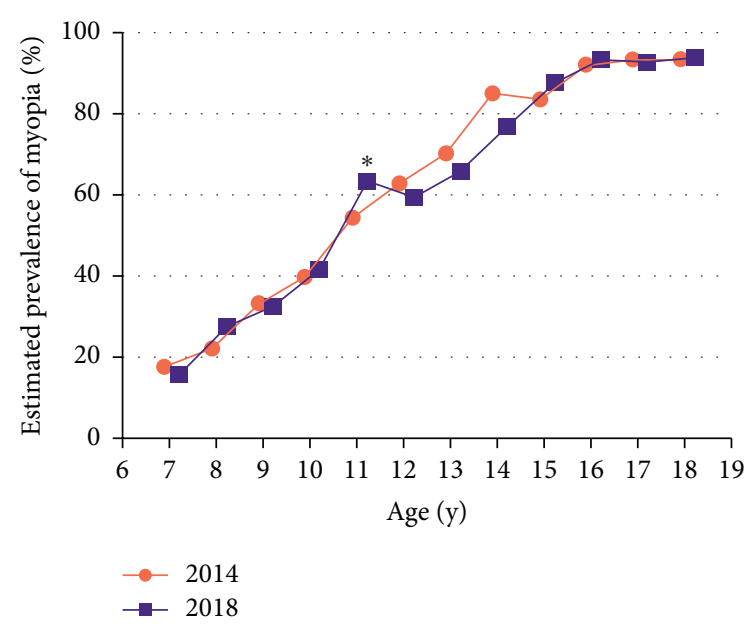

FIGURE 2: Trends of prevalence of myopia in children from 7- to 18year-olds in 2014 and 2018.

SE decreased from an average of $+1.05 \mathrm{D}$ at 7 -year-olds to $-4.02 D$ at 18 -year-olds in Shanghai, with a decrease of approximately $0.46 \mathrm{D} /$ year. He et al. [28] found that SE decreased from a median of $+1.25 \mathrm{D}$ of 5 -year-olds to -1.50 $D$ at 15-year-olds in Guangzhou, with a decrease of approximately $0.28 \mathrm{D} /$ year. Thorn et al. [29] found that SE decreased from $-0.36 \pm 1.19 D$ in key schools and $-0.38 \pm 1.14 D$ in nonkey schools in grade 1 (approximately 7-year-olds) to $-2.12 \pm 1.79 \mathrm{D}$ and $-1.41 \pm 1.56 \mathrm{D}$ in grade 6 (approximately 12-year-olds) in Wenzhou, with a decrease of approximately $0.35 \mathrm{D} /$ year and $0.21 \mathrm{D} /$ year, respectively. He et al. [30] found that mean SE was $-0.17 D$ to $-0.33 D$ for boys at $13-17$-year-olds and $-0.38 D$ to $-1.25 D$ for girls in Yangjiang city, Guangdong, with a decrease of approximately $0.04 \mathrm{D} /$ year and $0.22 \mathrm{D} /$ year, respectively. However, the differences in SE and its decrease rate still existed among different studies. For example, the mean SE of $-3.43 \mathrm{D}$ at $15-$ year-olds in 2018 in this study was smaller than $-2.29 \mathrm{D}$ in Xiong and coworkers' study [25] and $-1.50 \mathrm{D}$ in $\mathrm{He}$ and coworkers' study [28]. Except the reasons for the differences of SE similar to AL, refraction measured with cycloplegia or not was a potential cause because of approximately $0.50 \mathrm{D}$ in refraction after and before cycloplegia in children in our previous study [15].

In this study, the prevalence of myopia and high myopia increased with age in both 2014 and 2018, which was higher than other studies. The prevalence of myopia increased from approximately $5 \%$ at 7 -year-olds to $15 \%$ at 15 -year-olds in Chile [31]. The prevalence of myopia increased from $1.76 \%$ at 4 -year-olds children to $84.6 \%$ at 17 -year-olds in Shandong [32]. Pan et al. [33] found that the prevalence of myopia and high myopia was higher in grade 7 students (approximately 13-year-olds, $29.4 \%$ and $0.4 \%$, respectively) compared with grade 1 students (approximately 7 -year-olds, $2.4 \%$ and $0.1 \%$, respectively). The present study showed that the prevalence of high myopia started to increase at the age of 11-13-yearolds (Figure 3). The most important predictor of high myopia in myopic children was age of myopia onset or duration of myopia progression [34]. The younger the children are at myopia onset, the more likely they are to develop into high myopia, who may suffer from sightthreatening complications. In this study, both criteria of high myopia based on SE and AL were used to obtain more comprehensive evaluation, and the higher prevalence of high myopia defined by AL was observed compared to that defined by SE.

The most interesting findings of this study were that the prevalence of myopia and high myopia decreased, as well as the decrease of AL and increase of SE in 2018 compared to 2014, which may be due to the younger children in 2018 than in 2014. These were different from other studies. The comparisons of prevalence of myopia in Taiwan children showed that it increased from $5.8 \%$ in 1983 to $21 \%$ in 2000 at 7 -year-olds, $36.7 \%$ to $61 \%$ at 12 -year-olds, $64.2 \%$ to $81 \%$ at 15 -year-olds, and $74 \%$ to $84 \%$ at $16-18$-year-olds, respectively, and the prevalence of high myopia from $10.9 \%$ to $21 \%$ at 18-year-olds [35]. In Spain, the prevalence of myopia and high myopia in children at 5-7-year-olds slightly increased from $17 \%$ in 2016 to $19 \%$ in 2017 and from $1.7 \%$ to $1.9 \%$, 


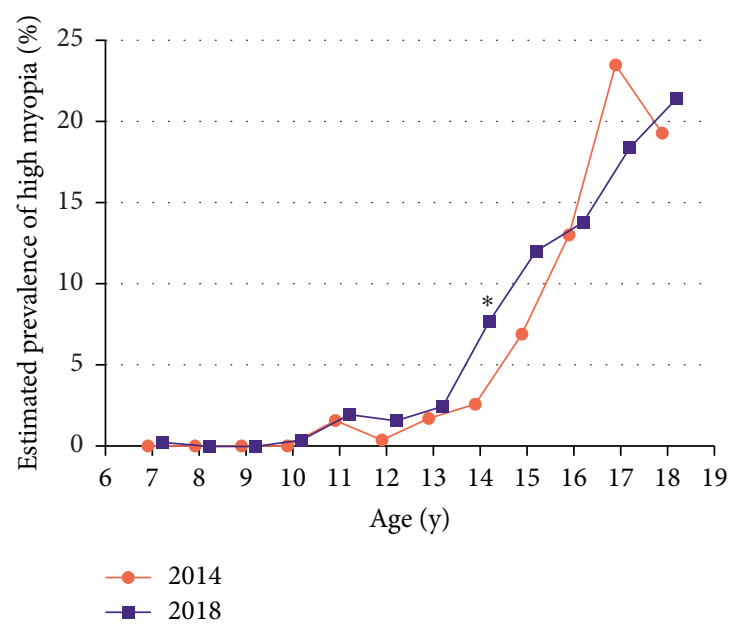

(a)

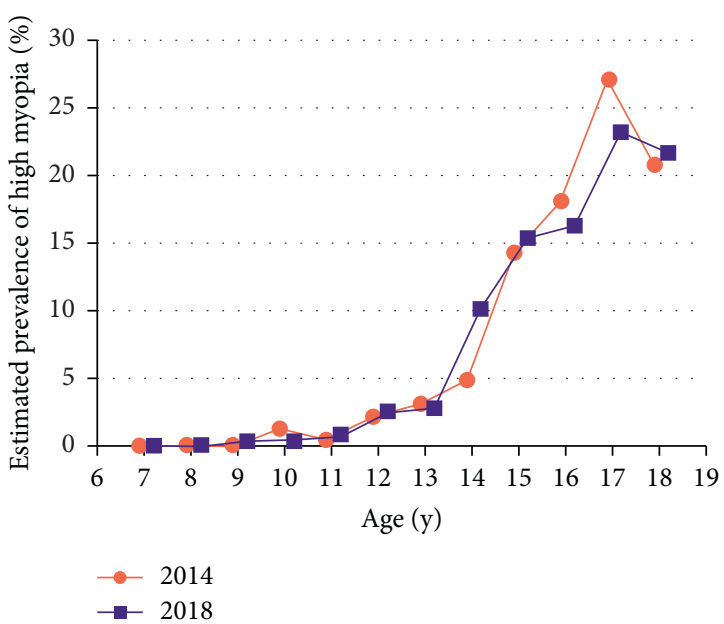

(b)

FIGURE 3: Trends of prevalence of high myopia defined by $\mathrm{SE} \leq-6.00 \mathrm{D}$ (a) and by AL $>26 \mathrm{~mm}$ (b) in children from 7- to 18-year-olds in 2014 and 2018.

respectively [36]. In the US, the prevalence of myopia increased from $12.0 \%$ in $1971-1972$ to $31.2 \%$ in $1999-2004$ in children at 12-17-year-olds [37]. It is predicted that the global prevalence of myopia and high myopia will increase from $23 \%$ in 2000 to $50 \%$ in 2050 and from $3 \%$ to $10 \%$ [15]. This discrepancy may be due to the improvement of myopia prevention and control concept worldwide, and the emphasis on myopia in China in recent years [38]. In addition, shorter AL in 2018 compared to 2014 could be explained by those boys who had shorter AL in 2018 compared to 2014. Prospective longitudinal studies with longer time frame are warranted to evaluate the change in $\mathrm{AL}, \mathrm{SE}$, and the prevalence of myopia and high myopia in the future.

In our study, children spent less time outdoors and more time of study in 2018 than in 2014. Previous studies have shown a protective effect of outdoor activities on myopia onset $[39,40]$. Although the meta-analyses by Deng and Pang [41] suggested that the effect of time outdoors on AL and refractive error was very small, and time outdoors could retard myopic progression in all initially nonmyopic children. So, it is very critical for teachers and parents or guardians of children to emphasize the importance of outdoor activities, especially nonmyopic children.

There are several limitations in this study. First, AL was analyzed in only 3 schools with a small sample size, which could not be a representative of the whole of Shanghai. Further studies with larger sample size are warranted. Second, SE was determined by noncycloplegic autorefraction, which may lead to an overestimation of myopia. Our previous study showed that there was less than $-0.50 \mathrm{D}$ of difference between cycloplegic and noncycloplegic refraction among 7-16-year-olds children [15]. Furthermore, the procedure without cycloplegia was simple and timesaving and could not affect the later learning and writing of children.

In conclusion, the present study demonstrates normative growth values for AL and SE in Shanghai children at the age of 7-18-year-olds, as well as the age-specific prevalence of myopia and high myopia. Less time outdoors and more time of study are also observed in Shanghai children. Children should be encouraged to participate in outdoor activities, especially nonmyopia children. Longitudinal studies are needed to evaluate the trends of AL, SE, prevalence of myopia and high myopia, and associated risk factors in the future.

\section{Data Availability}

The dataset used to support this study is available from the Dryad repository, https://doi.org/10.5061/dryad.c2fqz615c.

\section{Conflicts of Interest}

The authors declare that they have no conflicts of interest.

\section{Acknowledgments}

This study was supported in part by the Project of Shanghai Science and Technology (Grant nos. 17411950200, 17411950203, and 17ZR1404200) and the Project of Shanghai Health and Family Planning Committee (Grant no. 20174Y0177).

\section{References}

[1] I. G. Morgan, K. Ohno-Matsui, and S. M. Saw, "Myopia," The Lancet, vol. 379, no. 9827, pp. 1739-1748, 2012.

[2] I. G. Morgan, A. N. French, R. S. Ashby et al., "The epidemics of myopia: aetiology and prevention," Progress In Retinal And Eye Research, vol. 62, pp. 134-149, 2018.

[3] E. Dolgin, "The myopia boom," Nature, vol. 519, no. 7543, pp. 276-278, 2015.

[4] M. Scheiman, J. Gwiazda, Q. Zhang et al., "Longitudinal changes in corneal curvature and its relationship to axial length in the correction of myopia evaluation trial (COMET) cohort," Journal of Optometry, vol. 9, no. 1, pp. 13-21, 2016. 
[5] D. K. VanderVeen, R. T. Kraker, S. L. Pineles et al., "Use of orthokeratology for the prevention of myopic progression in children," Ophthalmology, vol. 126, no. 4, pp. 623-636, 2019.

[6] A. Meduri, L. Scorolli, S. Scalinci et al., "Effect of the combination of basic fibroblast growth factor and cysteine on corneal epithelial healing after photorefractive keratectomy in patients affected by myopia," Indian Journal of Ophthalmology, vol. 62, no. 4, pp. 424-428, 2014.

[7] M. C. Arbelaez, C. Vidal, and S. Arba Mosquera, "Comparison of LASEK and LASIK with thin and ultrathin flaps after excimer laser ablation with the SCHWIND aspheric ablation profile," Journal of Refractive Surgery, vol. 27, no. 1, pp. 38-48, 2011.

[8] M. Li, M. Li, Y. Chen et al., "Five-year results of small incision lenticule extraction (SMILE) and femtosecond laser LASIK(FS-LASIK) for myopia," Acta Ophthalmologica, vol. 97, no. 3, pp. e373-e380, 2019.

[9] Z. Yan, H. Miao, F. Zhao et al., "Two-year outcomes of visian implantable collamer lens with a central hole for correcting high myopia," Journal of Ophthalmology, vol. 2018, Article ID 8678352, 13 pages, 2018.

[10] K. Ohno-Matsui, T. Y. Y. Lai, C.-C. Lai, and C. M. G. Cheung, "Updates of pathologic myopia," Progress in Retinal and Eye Research, vol. 52, pp. 156-187, 2016.

[11] Y. Ikuno, "Overview of the complications of high myopia," Retina, vol. 37, no. 12, pp. 2347-2351, 2017.

[12] H. Senra, F. Barbosa, P. Ferreira et al., "Psychologic adjustment to irreversible vision loss in adults," Ophthalmology, vol. 122, no. 4, pp. 851-861, 2015.

[13] Z. Liu, D. Wu, J. Huang et al., "Visual impairment, but not hearing impairment, is independently associated with lower subjective well-being among individuals over 95 years of age: a population-based study," Archives of Gerontology And Geriatrics, vol. 62, pp. 30-35, 2016.

[14] C. W. Pan, M. Dirani, C. Y. Cheng, T. Y. Wong, and S. M. Saw, "The age-specific prevalence of myopia in asia," Optometry and Vision Science, vol. 92, no. 3, pp. 258-266, 2015.

[15] B. A. Holden, T. R. Fricke, D. A. Wilson et al., "Global prevalence of myopia and high myopia and temporal trends from 2000 through 2050," Ophthalmology, vol. 123, no. 5, pp. 1036-1042, 2016.

[16] S. K. Wang, Y. Guo, C. Liao et al., "Incidence of and factors associated with myopia and high myopia in Chinese children, based on refraction without cycloplegia," JAMA Ophthalmology, vol. 136, no. 9, pp. 1017-1024, 2018.

[17] E. Yotsukura, H. Torii, M. Inokuchi et al., "Current prevalence of myopia and association of myopia with environmental factors among schoolchildren in Japan," JAMA Ophthalmology, vol. 137, Article ID e193103, 2019.

[18] J. W. L. Tideman, J. R. Polling, V. W. V. Jaddoe, J. R. Vingerling, and C. C. W. Klaver, "Environmental risk factors can reduce axial length elongation and myopia incidence in 6- to 9-year-old children," Ophthalmology, vol. 126, no. 1, pp. 127-136, 2019.

[19] I. G. Morgan, A. N. French, and K. A. Rose, "Intense schooling linked to myopia," BMJ, vol. 361, p. k2248, 2018.

[20] E. Mountjoy, N. M. Davies, D. Plotnikov et al., "Education and myopia: assessing the direction of causality by mendelian randomisation," BMJ (Clinical Research Ed.vol. 361, Article ID k2022, 2018.

[21] T. Li, X. Zhou, X. Chen, H. Qi, and Q. Gao, "Refractive error in Chinese preschool children," Eye \& Contact Lens: Science \& Clinical Practice, vol. 45, no. 3, pp. 182-187, 2019.
[22] T. Li, B. Jiang, and X. Zhou, "Axial length elongation in primary school-age children: a 3-year cohort study in Shanghai," BMJ Open, vol. 9, no. 10, Article ID e29896, 2019.

[23] D. I. Flitcroft, M. He, J. B. Jonas et al., "Imi-defining and classifying myopia: a proposed set of standards for clinical and epidemiologic studies," Investigative Opthalmology \& Visual Science, vol. 60, no. 3, pp. M20-M30, 2019.

[24] S. M. Saw, S. Matsumura, and Q. V. Hoang, "Prevention and management of myopia and myopic pathology," Investigative Opthalmology \& Visual Science, vol. 60, no. 2, pp. 488-499, 2019.

[25] S. Xiong, B. Zhang, Y. Hong et al., "The associations of lens power with age and axial length in healthy Chinese children and adolescents aged 6 to 18 years," Investigative Opthalmology \& Visual Science, vol. 58, no. 13, pp. 5849-5855, 2017.

[26] T. L. Lu, J. F. Wu, X. Ye et al., "Axial length and associated factors in children: the Shandong children eye study," Ophthalmologica, vol. 235, no. 2, pp. 78-86, 2016.

[27] J. W. L. Tideman, J. R. Polling, J. R. Vingerling et al., "Axial length growth and the risk of developing myopia in European children," Acta Ophthalmologica, vol. 96, no. 3, pp. 301-309, 2018.

[28] M. He, J. Zeng, Y. Liu, J. Xu, G. P. Pokharel, and L. B. Ellwein, "Refractive error and visual impairment in urban children in southern China," Investigative Opthalmology \& Visual Science, vol. 45, no. 3, pp. 793-799, 2004.

[29] F. Thorn, J. Chen, C. Li et al., "Refractive status and prevalence of myopia among Chinese primary school students," Clinical and Experimental Optometry, vol. 103, 2019.

[30] M. He, W. Huang, Y. Zheng, L. Huang, and L. B. Ellwein, "Refractive error and visual impairment in school children in rural southern China," Ophthalmology, vol. 114, no. 2, pp. 374-382, 2007.

[31] E. Maul, S. Barroso, S. R. Munoz, R. D. Sperduto, and L. B. Ellwein, "Refractive error study in children: results from La Florida, Chile," American Journal of Ophthalmology, vol. 129, no. 4, pp. 445-454, 2000.

[32] J. F. Wu, H. S. Bi, S. M. Wang et al., "Refractive error, visual acuity and causes of vision loss in children in Shandong, China the shandong children eye study," PLoS One, vol. 8, no. 12, Article ID e82763, 2013.

[33] C. Pan, R. Wu, J. Li, and H. Zhong, "Low prevalence of myopia among school children in rural China," BMC Ophthalmology, vol. 18, no. 1, p. 140, 2018.

[34] S. Y. L. Chua, C. Sabanayagam, Y.-B. Cheung et al., "Age of onset of myopia predicts risk of high myopia in later childhood in myopic Singapore children," Ophthalmic and Physiological Optics, vol. 36, no. 4, pp. 388-394, 2016.

[35] L. L. Lin, Y. F. Shih, C. K. Hsiao, and C. J. Chen, "Prevalence of myopia in Taiwanese schoolchildren: 1983 to 2000," Ann Acad Med Singapore, vol. 33, no. 1, pp. 27-33, 2004.

[36] C. C. Alvarez-Peregrina, M. A. M. A. Sanchez-Tena, C. C. Martinez-Perez, and C. C. Villa-Collar, "Prevalence and risk factors of myopia in Spain," Journal of Ophthalmology, vol. 2019, Article ID 3419576, 7 pages, 2019.

[37] S. Vitale, R. D. Sperduto, and F. R. Ferris, "Increased prevalence of myopia in the United States between 1971-1972 and 1999-2004," Archives of Ophthalmology, vol. 127, no. 12, pp. 1632-1639, 2009.

[38] C. Jan, L. Li, L. Keay, R. S. Stafford, N. Congdon, and I. Morgan, "Prevention of myopia, China," Bulletin of the World Health Organization, vol. 98, no. 6, pp. 435-437, 2020.

[39] C.-C. Hsu, N. Huang, P.-Y. Lin et al., "Prevalence and risk factors for myopia in second-grade primary school children in 
Taipei: a population-based study," Journal of the Chinese Medical Association, vol. 79, no. 11, pp. 625-632, 2016.

[40] P.-C. Wu, C.-T. Chen, K.-K. Lin et al., "Myopia prevention and outdoor light intensity in a school-based cluster randomized trial," Ophthalmology, vol. 125, no. 8, pp. 1239-1250, 2018.

[41] L. Deng and Y. Pang, "Effect of outdoor activities in myopia control," Optometry and Vision Science, vol. 96, no. 4, pp. 276-282, 2019. 